\title{
Adherence to antidepressant therapy and mortality rates in ischaemic heart disease: cohort study
}

\author{
Amir Krivoy, Ran D. Balicer, Becca Feldman, Moshe Hoshen, Gil Zalsman, Abraham Weizman \\ and Gal Shoval
}

\section{Background}

The use of antidepressant drugs in patients with ischaemic heart disease (IHD) has been debated owing to scarcity of data and conflicting results regarding the effect of these drugs on mortality.

\section{Aims \\ To evaluate the association between adherence to antidepressant therapy and all-cause mortality in a population-based cohort of patients with IHD.}

\author{
Method \\ A total of 63437 patients with IHD who purchased \\ antidepressants at least once during the years 2008-2011 \\ were retrospectively followed for all-cause mortality over 4 \\ years. Adherence was measured as a ratio between claimed \\ and prescribed durations of medication and modelled as \\ non-adherence $(<20 \%)$, poor $(20-50 \%)$, moderate $(50-80 \%)$ \\ and good (>80\%). We used multivariable survival analyses
}

adjusted for demographic and clinical variables that may affect mortality.

\section{Results}

The moderate and good adherence groups had significantly reduced adjusted mortality hazard ratios of $0.83(95 \% \mathrm{Cl}$ $0.78-0.88)$ and $0.86(95 \% \mathrm{Cl} 0.82-0.90)$ respectively, compared with the non-adherence group.

\section{Conclusions}

Adherence to antidepressant pharmacotherapy is associated with reduced all-cause mortality in a population-based large sample cohort of patients with IHD. Physicians and health policy decision-makers should step up their efforts to sustain and enhance these patients' adherence to their antidepressant regimen

\section{Declaration of interest}

None.
Substantial evidence indicates that major depressive disorder is an independent risk factor for poor prognosis, including increased mortality, among patients with ischaemic heart disease (IHD). ${ }^{1}$ The mainstay treatment for depression is antidepressant drugs, which are shown to be effective also in the presence of other comorbid physical illnesses. ${ }^{2}$ However, the use of antidepressants in patients with cardiac disease, which was ranked first in terms of disability in the Global Burden of Disease Study, ${ }^{3}$ has been debated owing to conflicting results regarding their effect on mortality. Whereas some studies have shown that antidepressant use in patients with cardiac disease was associated with decreased mortality, ${ }^{4-6}$ other publications found no significant impact, ${ }^{7-9}$ and some demonstrated an increased mortality risk..$^{10-13}$ Only one study so far has addressed the relationship between adherence to antidepressant therapy and mortality in patients with cardiac disease. A 7-year follow-up study of 4037 patients with depression following myocardial infarction showed that patients with inadequately short duration of treatment for depression (less than 12 weeks of continuous antidepressant treatment) had a 3 -fold higher mortality risk compared with those sufficiently treated. ${ }^{14}$ However, this British study evaluated only the duration of continuous antidepressant use (termed persistence), not any other measures of inadequate adherence, such as irregular dosing and skipped doses - measures that are considered essential in a comprehensive assessment of medication adherence. ${ }^{15}$

Adherence to antidepressant therapy is critical to successful treatment outcomes. ${ }^{16}$ However, low adherence is commonly reported among people who are prescribed antidepressants, ${ }^{17-20}$ including patients with cardiac disease. ${ }^{14,21,22}$ Improvement of adherence to antidepressant therapy in the treatment of anxiety and depression is therefore a major public health concern and has been recognised by the World Health Organization (WHO) as an urgent need and a major area for intervention. ${ }^{23}$ Our primary objective was to evaluate the association between allcause mortality rates and adherence to antidepressants among a patient population with IHD. We predicted that adherence to antidepressants would be associated with lower mortality in these patients. We used data from the integrated medical records of Clalit Health Services (CHS), the largest health provider in Israel, which covers over 4 million patients from cradle to grave and includes demographic information, diagnoses from ambulatory services, family physicians, hospital admissions and specialists, drug prescriptions, laboratory test results and imaging. This enabled us to perform a cohort study with a sample size much larger than the total of all previously published cohorts of patients with cardiac disease maintained on antidepressants, and to include patients of all ages, for the first time investigating whether mortality is affected by adherence to antidepressant therapy.

\section{Method}

We retrospectively analysed the entire CHS patient population during the study period (1 January 2008 until 1 January 2012) at all ages $(n=4056700)$. We included all patients with at least one prescription for an antidepressant drug during the study period and a clinical diagnosis of IHD (ICD code 120-125) $(n=79711) .^{25}$ All antidepressant drugs approved by the Food and Drug Administration were included; extraction was based on the WHO Anatomical Therapeutic Chemical (ATC) code N06A. In the final analyses we included only data from those identified as antidepressant users, namely patients who purchased 
at least one prescription $(n=63437)$. Patients have a strong incentive to access primary care and obtain prescription medications within the CHS system because primary care visits are free of charge to members and medications included in the benefit package are subject to highly reduced co-payments (approximately US \$4-7 per month for most medications). Disadvantaged subpopulations are entitled to additional discounts. Access to the data warehouse and the analyses were approved for this study by the CHS review board.

\section{Primary outcome}

A record of death from any cause during the 4-year study period (produced by the Ministry for Interior Affairs) was the primary study outcome. Patients were followed up from entry to study (i.e. prescription of an antidepressant) until death or were censored at the end of the study period.

\section{Adherence}

The adherence measure was modelled on the basis of the concept of medication possession ratio (data from CHS pharmacies) with the addition of physician prescription data (derived from electronic medical records), as previously described in detail by us. $^{25}$ Study participation duration was calculated as the continuous period between the first and last antidepressant prescriptions. Adherence was defined as the period during which antidepressants were purchased (months) during follow-up, divided by study participation duration (months), expressed as a percentage. Thus it reflects adherence over the period for which antidepressants were known to be prescribed. Adherence (medication possession ratio) was calculated across all antidepressants used; switching between different antidepressant compounds was not taken into account. Patients with adherence below $20 \%$ were considered non-adherent, those with $20-50 \%$ adherence were considered poorly adherent, those with $50-80 \%$ were considered to have a moderate adherence and patients with adherence above $80 \%$ were considered to have good adherence.

Covariates. We collected the following sociodemographic and clinical variables at study entry: age (categorised into $0-18$, 19-24, 25-40, 41-64, 65-74, 75-84 and 85+ years), gender, socioeconomic status and self-reported smoking status (categorised as those who never smoked and those who smoked in the past or present). We extracted all available data on physical comorbidities (ICD-10 diagnoses). Based on these data, we calculated the Charlson Comorbidity Index (CCI), the most widely used clinical index for the evaluation of comorbidities. ${ }^{26}$ The CCI weighs 20 chronic conditions as predictors of 1-year relative risk of death, and is scored between 1 and 20 .

\section{Statistical analysis}

Statistical analysis was conducted using SPSS version 20 for Windows. We recorded descriptive statistics of sociodemographic factors, comorbidities and adherence levels across the total study population as well as across the four adherence level groups. Univariate analyses (logistic regression and Kaplan-Meier log rank) were used to assess the association between sociodemographic and clinical covariates and those who died or survived during the study period. The multivariable Cox proportional hazard regression model was used to assess the adjusted association between risk of death and adherence level of antidepressant medication, controlling for the confounders found to be significant in the univariate analysis (age, gender, physical comorbidities modelled as CCI, smoking status and socioeconomic status). We tested the assumptions of the proportional hazard model using $\log (-\log )$ plots. Hazard ratios
(HR) and their 95\% confidence intervals are reported, with a significance level of $P<0.05$. All analyses were two-tailed.

\section{Results}

Population characteristics and mortality rates are shown in Table 1. Almost three-quarters of the study population were over 65 years old; none was below 18 years old. The most prevalent comorbidities among the study population were hypertension $(84.8 \%)$, diabetes mellitus $(45.8 \%)$, chronic heart failure $(22.2 \%)$ and past stroke (23.3\%). The most frequent concomitantly used drugs were statins (86.2\%), acetylsalicylic acid (79.9\%) and anxiolytics (50.1\%). Men and women had similar unadjusted mortality rates $(19.5 \%$ and $18.7 \%$ respectively) and those with higher CCI scores were more likely to die $(41.7 \%$ for CCI $>5$ v. $11.9 \%$ for CCI $0-2, P<0.0001)$. The mean followup study period was 26.8 months (s.d. $=16$, range $1-47$, median 30 ); the mean follow-up time of survivors was 27.9 months $($ s.d. = 15.6) and mean time to death among those who died was 21.6 months $($ s.d. $=12)$.

\section{Adherence}

Thirty-six per cent of the study sample discontinued antidepressants within a month after prescription (i.e. they had only a single purchase) and $30.3 \%$ of the sample discontinued the drug after less than 6 months. Adherence distribution and variables across adherence levels are shown in Table 2. The adherence distribution indicated that $28.7 \%$ of those using antidepressants were non-adherent $(n=18212), 15.9 \%$ had poor adherence $(n=10094), 17.4 \%$ had moderate adherence $(n=11041)$ and $37.9 \%$ had good adherence $(n=24090)$.

\section{Univariate analysis}

Unadjusted analyses (Table 2) show that those with non-adherence had the lowest mortality rate $(13.8 \%)$ compared with the mortality

Table 1 All patients with ischaemic heart disease $(n=63437)$ characteristics and mortality rates

\begin{tabular}{|c|c|c|c|c|}
\hline & \multicolumn{2}{|c|}{ Population } & \multicolumn{2}{|c|}{ Mortality } \\
\hline & $n$ & (\%) & $n$ & (\%) \\
\hline Total & 63437 & (100) & 11789 & (19) \\
\hline \multicolumn{5}{|l|}{ Gender } \\
\hline Male & 31054 & (50) & 5877 & (19) \\
\hline Female & 32383 & (50) & 5912 & (18) \\
\hline \multicolumn{5}{|c|}{ Age at study entry, years } \\
\hline $18-24$ & 21 & $(0)$ & 0 & (0) \\
\hline 25-39 & 338 & $(0)$ & 4 & $(1)^{\star}$ \\
\hline $40-64$ & 14882 & (16) & 896 & $(6)^{*}$ \\
\hline $65-74$ & 15212 & (14) & 1935 & $(13)^{*}$ \\
\hline $75-84$ & 23489 & (24) & 5194 & $(22)^{*}$ \\
\hline$>85$ & 9495 & (13) & 3760 & $(40)$ \\
\hline \multicolumn{5}{|c|}{ Socioeconomic status } \\
\hline Low & 21388 & (34) & 3853 & (18) \\
\hline Moderate & 27765 & (44) & 5188 & $(19)^{*}$ \\
\hline High & 14174 & (22) & 2719 & $(19)^{*}$ \\
\hline \multicolumn{5}{|l|}{$\mathrm{CCl}$ score } \\
\hline 0-2 & 29846 & (47) & 3522 & $(12)$ \\
\hline $3-4$ & 17733 & (28) & 3385 & $(19)^{*}$ \\
\hline$>5$ & 15798 & (25) & 4872 & $(31)^{*}$ \\
\hline \multicolumn{5}{|l|}{ Smoking status } \\
\hline Never & 42228 & (67) & 8386 & (15) \\
\hline Past or current & 20385 & (33) & 3053 & $(20)^{*}$ \\
\hline $\begin{array}{l}\mathrm{CCl} \text {, Charlson Comorb } \\
{ }^{\star} P<0.05 \text {. }\end{array}$ & & & & \\
\hline
\end{tabular}




\begin{tabular}{|c|c|c|c|c|}
\hline & \multicolumn{4}{|c|}{ Adherence, $n(\%)$} \\
\hline & None $<20 \%$ & Poor $20-50 \%$ & Moderate $50-80 \%$ & Good $>80 \%$ \\
\hline \multicolumn{5}{|l|}{ Gender } \\
\hline Male & $9230(30)$ & $5023(16)$ & $5303(17)$ & $11498(37)$ \\
\hline Female & $8982(28)$ & $5071(16)$ & $5738(18)$ & 12592 (39) \\
\hline \multicolumn{5}{|c|}{ Age at study entry, years } \\
\hline $18-24$ & $9(43)$ & $4(19)$ & $4(19)$ & $4(19)$ \\
\hline $25-39$ & $156(46)$ & $66(20)$ & $55(16)$ & $61(18)$ \\
\hline $40-64$ & $5711(38)$ & $2543(17)$ & $2352(16)$ & $4276(29)$ \\
\hline $65-74$ & $4640(31)$ & 2469 (16) & $2658(17)$ & $5445(36)$ \\
\hline $75-84$ & $5645(24)$ & 3634 (15) & $4183(18)$ & $10027(43)$ \\
\hline $85+$ & $2051(22)$ & $1378(15)$ & 1789 (19) & $4277(45)$ \\
\hline \multicolumn{5}{|c|}{ Socioeconomic status } \\
\hline Low & 7142 (33) & 3679 (17) & $3583(17)$ & $6984(33)$ \\
\hline Moderate & $7708(28)$ & $4353(16)$ & 4878 (18) & 10826 (39) \\
\hline High & $3346(24)$ & 2047 (14) & $2554(18)$ & $6227(44)$ \\
\hline \multicolumn{5}{|l|}{ Smoking } \\
\hline Never & $11811(28)$ & $6742(16)$ & 7441 (18) & $16234(38)$ \\
\hline Ever & $6218(31)$ & $3258(16)$ & $3450(17)$ & 7459 (37) \\
\hline \multicolumn{5}{|l|}{$\mathrm{CCl}$ score } \\
\hline $0-2$ & 9092 (30) & $4786(16)$ & $5161(17)$ & $10807(36)$ \\
\hline $3-4$ & $4952(28)$ & 2794 (16) & 3131 (18) & 6856 (39) \\
\hline$>5$ & $4150(26)$ & 2504 (16) & $2744(17)$ & $6400(41)$ \\
\hline Mortalitya & $2513(13.8)$ & $1823(18.1)$ & 2137 (19.4) & $5316(22.1)$ \\
\hline Total & $18212(28.7)$ & 10094 (15.9) & 11041 (17.4) & 24090 (38) \\
\hline
\end{tabular}

rates of those with poor adherence $(18.1 \%)$, moderate adherence (19.4\%) or good adherence $(22.1 \% ; P<0.001$ for all). These statistically significant differences were corroborated by a univariate Kaplan-Meier model (results not shown). Older adults ( $>75$ years) were more likely to have good adherence than to be non-adherent $(43 \%$ v. $24 \% ; P<0.001$, Table 2). Furthermore, there were more severely physically ill patients $(\mathrm{CCI}>5)$ in the good adherence group compared with the non-adherence group (41\% v. 26\%; $P<0.001)$.

\section{Multivariable analysis}

The Cox proportional hazards model included mortality as the main outcome and variables significantly associated with mortality in the univariate analyses as covariates. Adjusted associations between adherence to antidepressant therapy and mortality rate appear in Fig. 1. The hazard ratios of mortality among the poor, moderate and good adherence groups were 0.97 (95\% CI $0.9-1.03), 0.83$ (95\% CI $0.78-0.88)$ and 0.86 (95\% CI $0.82-0.90)$ respectively compared with the non-adherence group. Using the same model, the adjusted HR for death among men was 1.27 (95\% CI 1.22-1.32) compared with women. Other predictors positively associated with mortality were age (25-39 years, $\mathrm{HR}=0.032,95 \%$ CI $0.01-0.09 ; 40-64$ years, $\mathrm{HR}=0.13$, $95 \%$ CI $0.12-0.14 ; 65-74$ years, $\mathrm{HR}=0.26,95 \%$ CI $0.25-0.28$; 75-84 years, $\mathrm{HR}=0.47,95 \%$ CI $0.45-0.49$; relative to age $85+$ years, all $P<0.001$ ); low socioeconomic status $(\mathrm{HR}=1.08,95 \%$ CI $1.03-1.14, P<0.003)$ and moderate socioeconomic status $(\mathrm{HR}=1.06,95 \%$ CI $1.01-1.11, P=0.019)$ compared with high socioeconomic status; and greater physical comorbidity (CCI score $0-2, \mathrm{HR}=0.39,95 \%$ CI $0.37-0.40, P<0.001$; CCI score $3-4, \mathrm{HR}=0.58,95 \%$ CI $0.55-0.60, P<0.001$ compared with CCI scores $5+$ ). Current or past smoking status ( $\mathrm{HR}=1.03,95 \%$ CI $0.98-1.08$, NS) compared with never smoked status was not significantly associated.

\section{Discussion}

The main novel finding of our large population-based cohort study of patients with IHD is that moderate and good adherence to antidepressant therapy is associated with a substantially decreased risk of death ( $17 \%$ and $14 \%$ respectively) compared with the non-adherence group. The results of the multivariate model analysis suggest that mortality is lower among patients with IHD with better adherence to antidepressants after adjusting for other confounding risk factors for mortality such as age, gender, socioeconomic status, smoking status and physical comorbidities.

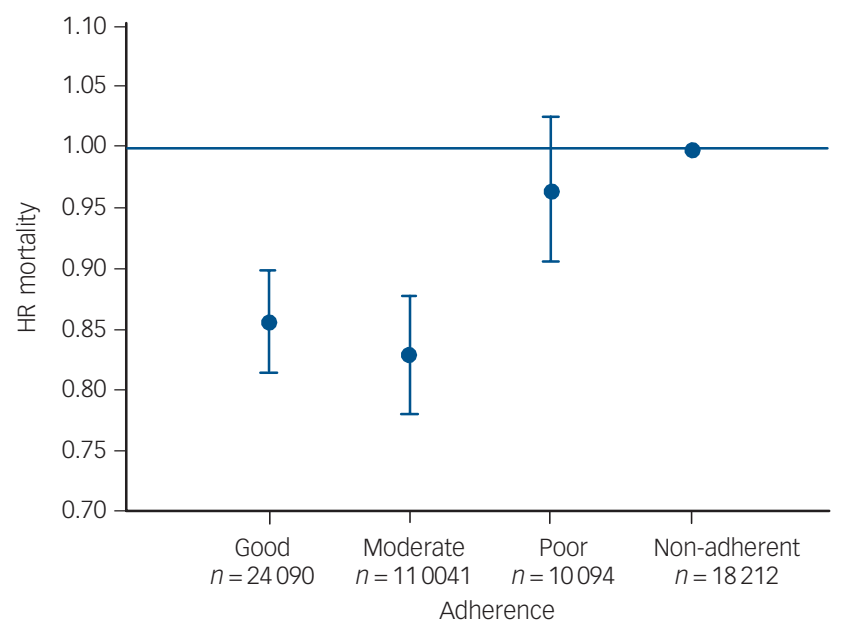

Fig. 1 Adjusted hazard ratios (HR) for mortality according to the level of adherence: good $>80 \%$, moderate $50-80 \%$, poor $20-50 \%$, non-adherence $<20 \%$ (reference). The variables were adjusted for gender, age, socioeconomic status, smoking and Charlson's comorbidity index. 
Interestingly, crude, unadjusted mortality rates increase with adherence levels $(13.8 \%, 18.1 \%, 19.4 \%$ and $22.1 \%$ at non-adherent, poor, moderate and good adherence levels respectively). The disparity between the unadjusted univariate results and the multivariable Cox analysis results may be explained by the strong confounding effects of gender, age, smoking, socioeconomic status and physical comorbidity (Table 2). Our crude data show that those with good adherence have more risk factors for mortality such as older age and more comorbid physical diseases, compared with those with lower adherence. Only by controlling for these substantially confounding risk factors, using the multivariate model analysis, could the specific unbiased contribution of adherence to mortality be revealed.

Our findings are consistent with the only report to date on the detrimental effect of suboptimal longitudinal treatment with antidepressants. It was found that less than 12 weeks of continuous antidepressant treatment, a crude proxy variable for adherence, was associated with a three-fold mortality risk. ${ }^{14}$ Two other, related studies examined the association between the use of antidepressants as a dichotomous variable (i.e. use or non-use rather than measuring adherence over time) and all-cause mortality among patients with IHD. The studies yielded inconsistent results: a prospective US study of 361 patients with acute coronary syndrome and depression showed a reduced mortality risk of 0.42 in those using antidepressants $v$. non-users, ${ }^{5}$ but the Enhancing Recovery in Coronary Heart Disease Patients (ENRICHD) randomised trial of 2481 post-infarction patients reported no significant difference between those who used antidepressants and those who did not. ${ }^{11}$ Our study broadened this initial observation to the population of patients with all types of IHD in a much larger cohort of 63437 patients using antidepressants (regardless of depression status) of all ages.

These conflicting results regarding the association of antidepressants with survival reflect the same debate regarding the effect of these medications in the general population and other clinical subpopulations. Whereas some reports indicated that antidepressant usage may decrease all-cause mortality risk, ${ }^{21,27,28}$ several other studies demonstrated increased mortality among patients taking antidepressants. ${ }^{29-33}$ These limited and contradictory findings, reported in relatively small samples adjusted for diverse covariates, further stress the importance of the findings from our study, which add in the parameter of antidepressant adherence in this high-risk IHD patient population. Our findings may also shed light on the clinically important question of whether even poor (20-50\%) adherence to antidepressant therapy has any benefit over non-adherence $(<20 \%)$ in terms of survival. This issue has not been addressed previously, although clinicians often encounter patients who are poorly adherent to treatment ( $16 \%$ of this cohort). The results of our analysis (Fig. 1) indicate that this low level of adherence $(20-50 \%)$ is not effective in decreasing mortality compared with the reference non-adherent group.

Several mechanisms may underlie the association between adherence to antidepressant drug treatment and survival in patients with IHD. A number of cardiovascular medications were previously shown to reduce mortality. ${ }^{34}$ It is therefore possible that the reduced mortality associated with adherence to antidepressants could be operating, at least to some extent, through adherence to medication with cardiovascular drugs rather than antidepressants. Moreover, good adherence to antidepressant drug therapy attenuates depression and anxiety and may thus promote adherence to cardiac medications. ${ }^{5}$ Depressive and anxiety disorders are very prevalent among patients with cardiovascular disease, which in turn lead to the aggravation of IHD, ${ }^{35}$ as well as other comorbid physical disorders. ${ }^{21,36}$ This finding might be the consequence of a decrease in adherence to cardiac, antihypertensive and other medications for comorbid diseases in this population compared with non-depressed patients, ${ }^{35,37}$ as suggested by preliminary reports of small-scale studies. ${ }^{5,33}$ Another suggested explanation is that depression is associated with neglectful health behaviour, which might be improved by antidepressant usage. ${ }^{38}$ Antidepressant adherence is presumably associated with more mindful health behaviours (e.g. physical activity and healthy nutrition) which might mediate the link between antidepressant adherence and increased survival. If so, this explanation might also underlie the effect of antidepressant adherence on survival in patients with IHD demonstrated in our study. However, these hypotheses remain to be tested in future studies.

\section{Study limitations}

The findings of this pharmacoepidemiologic study should be interpreted in light of its limitations. The analyses did not include data regarding the psychiatric diagnoses associated with the need for antidepressant prescription, as mental health diagnosis data in the database were incomplete. Additionally, data on causes of death were also not available, and thus we were unable to discriminate between deaths due to IHD, suicide and other causes. It is noteworthy, however, that suicide is not the primary cause of death even among people with depression and anxiety. ${ }^{39}$ Therefore, we considered all-cause mortality a preferable outcome measure to evaluate the long-term global impact of antidepressant medication adherence on the survival of patients with IHD. Another possible limitation could be related to our assumption that antidepressants are prescribed by the physician only during the time they are clinically justified. However, the denominator may be misleading if patients have only had discrete short episodes of depression separated by a long duration of remission, which could lead to misclassification of low adherence. Finally, unmeasured variables, such as obesity and physical inactivity, could have also confounded our findings.

\section{Study strengths}

Our study has several key strengths. Our measure of adherence was shown to be reliable. ${ }^{25}$ The large sample size $(n=63437)$ encompassed a nationwide sample of all patients with IHD who used antidepressants in the CHS database without an age limitation. Therefore, our findings are generalisable to the general population. Moreover, the Israeli healthcare delivery system is similar to those in other Western countries and thus our findings are also generalisable to these settings. The 4-year follow-up period was long enough to evaluate mortality as an outcome measure, while permitting a large person-time denominator and establishment of causal pathways.

\section{Implications}

Our 4-year follow-up study is the first to demonstrate the inverse association between adherence to antidepressants and all-cause mortality in a general-population-based, large cohort of patients with IHD. Even though the standardised effect size of antidepressants may be lower than previously thought, ${ }^{40}$ the beneficial effect of adherence to antidepressants may be particularly important in the high-risk population of patients with IHD. We suggest that cardiologists, psychiatrists and primary care physicians should step up their efforts to sustain and enhance their patients' adherence to antidepressant drug treatment, as it may be associated with increased life expectancy. Suboptimal duration and inadequate dosing of antidepressant treatment increase the risk of relapse, chronicity and - as shown here - 
mortality. The low adherent behaviour of patients with IHD, as found in the current study, is of considerable clinical and public health concern. This study contributes new data for health policy decision-makers to increase their awareness concerning the association between adherence to antidepressants and survival in patients with cardiac disease.

Amir Krivoy, MD, Clalit Research Institute, Tel Aviv, Geha Mental Health Center, Petah Tiqva, and Sackler Faculty of Medicine, Tel Aviv University, Tel Aviv; Ran D. Balicer, MD, PhD, Clalit Research Institute, Tel Aviv, Public Health Department, Faculty of Health Sciences, Ben-Gurion University, Beer-Sheva; Becca Feldman, PhD, Moshe Hoshen, PhD, Clalit Research Institute, Tel Aviv, Israel; Gil Zalsman, MD, MHA, Geha Mental Health Center, Petah Tiqva, Sackler Faculty of Medicine, Tel Aviv University, Tel Aviv, Israel, and Division of Molecular Imaging and Neuropathology, Department of Psychiatry, Columbia University, New York, USA; Abraham Weizman MD, Geha Mental Health Center, Petah Tiqva, Felsenstein Medical Research Center Sackler Faculty of Medicine, Tel Aviv University, Tel Aviv; Gal Shoval, MD, Clalit Research Institute, Tel Aviv, Geha Mental Health Center, Petah Tiqva, and Sackler Faculty of Medicine, Tel Aviv University, Tel Aviv, Israel

Correspondence: Dr Amir Krivoy, Deputy Director, Psychiatry Ward, Geha Mental Health Center, PO Box 102, Petah Tiqva 49 100, Israel. Email akrivoy@clalit.org.il

First received 17 Apr 2014, final revision 19 Aug 2014, accepted 15 Sep 2014

\section{References}

1 McGovern PG, Pankow JS, Shahar E, Doliszny KM, Folsom AR, Blackburn H et al. Recent trends in acute coronary heart disease - mortality, morbidity, medical care, and risk factors. The Minnesota Heart Survey Investigators. N Engl J Med 1996; 334: 884-90.

2 Rayner L, Price A, Evans A, Valsraj K, Higginson IJ, Hotopf M. Antidepressants for depression in physically ill people. Cochrane Database Syst Rev 2010; 3 CD007503.

3 Murray CJ, Vos T, Lozano R, Naghavi M, Flaxman AD, Michaud C, et al. Disability-adjusted life years (DALYS) for 291 diseases and injuries in 21 regions, 1990-2010: a systematic analysis for the Global Burden of Disease Study 2010. Lancet 2012; 380: 2197-223.

4 Enriched Investigators. Enhancing recovery in coronary heart disease patients (ENRICHD): study design and methods. The ENRICHD investigators. Am Heart J 2000; 139: 1-9.

5 Glassman AH, Bigger JT, Gaffney M. Psychiatric characteristics associated with long-term mortality among 361 patients having an acute coronary syndrome and major depression: seven-year follow-up of SADHART participants. Arch Gen Psychiatry 2009; 66: 1022-9.

6 Roose SP, Laghrissi-Thode F, Kennedy JS, Nelson JC, Bigger JT, Pollock BG, et al. Comparison of paroxetine and nortriptyline in depressed patients with ischemic heart disease. JAMA 1998; 279: 287-91.

7 Cohen HW, Gibson G, Alderman MH. Excess risk of myocardial infarction in patients treated with antidepressant medications: association with use of tricyclic agents. Am J Med 2000; 108: 2-8.

8 Frasure-Smith N, Lesperance F, Talajic M. Depression following myocardial infarction. Impact on 6-month survival. JAMA 1993; 270: 1819-25.

9 Meier CR, Schlienger RG, Jick $\mathrm{H}$. Use of selective serotonin reuptake inhibitors and risk of developing first-time acute myocardial infarction. Br J Clin Pharmacol 2001; 52: 179-84.

10 Ahern DK, Gorkin L, Anderson JL, Tierney C, Hallstrom A, Ewart C, et al. Biobehavioral variables and mortality or cardiac arrest in the Cardiac Arrhythmia Pilot Study (CAPS). Am J Cardiol 1990; 66: 59-62.

11 Berkman LF, Blumenthal J, Burg M, Carney RM, Catellier D, Cowan MJ, et al. Effects of treating depression and low perceived social support on clinical events after myocardial infarction: the Enhancing Recovery in Coronary Heart Disease Patients (ENRICHD) Randomized Trial. JAMA 2003; 289: 3106-16.

12 Bush DE, Ziegelstein RC, Tayback M, Richter D, Stevens S, Zahalsky H, et al. Even minimal symptoms of depression increase mortality risk after acute myocardial infarction. Am J Cardiol 2001; 88: 337-41.

13 O'Connor CM, Jiang W, Kuchibhatla M, Mehta RH, Clary GL, Cuffe MS, et al. Antidepressant use, depression, and survival in patients with heart failure. Arch Intern Med 2008; 168: 2232-7.

14 Scherrer JF, Chrusciel T, Garfield LD, Freedland KE, Carney RM, Hauptman PJ et al. Treatment-resistant and insufficiently treated depression and all-cause mortality following myocardial infarction. Br J Psychiatry 2012; 200: 137-42.

15 Chong WW, Aslani P, Chen TF. Effectiveness of interventions to improve antidepressant medication adherence: a systematic review. Int J Clin Pract 2011; 65: 954-75.
16 Geddes JR, Carney SM, Davies C, Furukawa TA, Kupfer DJ, Frank E, et al. Relapse prevention with antidepressant drug treatment in depressive disorders: a systematic review. Lancet 2003; 361: 653-61.

17 Bambauer KZ, Adams AS, Zhang F, Minkoff N, Grande A, Weisblatt R, et al. Physician alerts to increase antidepressant adherence: fax or fiction? Arch Intern Med 2006; 166: 498-504.

18 Hunot VM, Horne R, Leese MN, Churchill RC. A cohort study of adherence to antidepressants in primary care: the influence of antidepressant concerns and treatment preferences. Prim Care Companion J Clin Psychiatry 2007; 9 91-9.

19 Olfson M, Marcus SC, Tedeschi M, Wan GJ. Continuity of antidepressant treatment for adults with depression in the United States. Am J Psychiatry 2006; 163: 101-8

20 Sawada N, Uchida H, Suzuki T, Watanabe K, Kikuchi T, Handa T, et al. Persistence and compliance to antidepressant treatment in patients with depression: a chart review. BMC Psychiatry 2009; 9: 38.

21 Scherrer JF, Garfield LD, Lustman PJ, Hauptman PJ, Chrusciel T, Zeringue A, et al. Antidepressant drug compliance: reduced risk of $\mathrm{Ml}$ and mortality in depressed patients. Am J Med 2011; 124: 318-24.

22 Ziegelstein RC. Depression in patients recovering from a myocardial infarction. JAMA 2001; 286: 1621-7.

23 World Health Organization. Adherence to Long Term Therapies: Evidence for Action. WHO, 2003.

24 World Health Organization. The ICD-10 Classification of Mental and Behavioural Disorders: Clinical Descriptions and Diagnostic Guidelines. WHO, 1992.

25 Singer SR, Hoshen $M$, Shadmi E, Leibowitz M, Flaks-Manov N, Bitterman $H$, et al. EMR-based medication adherence metric markedly enhances identification of nonadherent patients. Am J Manag Care 2012; 18: e372-7.

26 Charlson ME, Pompei P, Ales KL, MacKenzie CR. A new method of classifying prognostic comorbidity in longitudinal studies: development and validation. J Chronic Dis 1987; 40: 373-83.

27 Gallo JJ, Bogner HR, Morales KH, Post EP, Lin JY, Bruce ML. The effect of a primary care practice-based depression intervention on mortality in older adults: a randomized trial. Ann Intern Med 2007; 146: 689-98.

28 Tiihonen J, Lonnqvist J, Wahlbeck K, Klaukka T, Tanskanen A, Haukka J. Antidepressants and the risk of suicide, attempted suicide, and overall mortality in a nationwide cohort. Arch Gen Psychiatry 2006; 63: 1358-67.

29 Almeida OP, Alfonso H, Hankey GJ, Flicker L. Depression, antidepressant use and mortality in later life: the Health In Men Study. PLOS One 2010; 5 : e11266.

30 Bingefors K, Isacson D, Knorring LV, Smedby B, Wicknertz K. Antidepressanttreated patients in ambulatory care. Mortality during a nine-year period after first treatment. Br J Psychiatry 1996; 169: 647-54.

31 Coupland C, Dhiman P, Morriss R, Arthur A, Barton G, Hippisley-Cox J. Antidepressant use and risk of adverse outcomes in older people: population based cohort study. BMJ 2011; 343: d4551.

32 Ryan J, Carriere I, Ritchie K, Stewart R, Toulemonde G, Dartigues JF, et al. Late-life depression and mortality: influence of gender and antidepressant use. Br J Psychiatry 2008; 192: 12-8.

33 Sundell KA, Gissler M, Petzold M, Waern M. Antidepressant utilization patterns and mortality in Swedish men and women aged 20-34 years. Eur J Clin Pharmacol 2011; 67: 169-78.

34 Selim AJ, Fincke BG, Rogers WH, Qian S, Selim BJ, Kazis LE. Guidelinerecommended medications: variation across Medicare Advantage plans and associated mortality. J Manag Care Pharm 2013; 19: 132-8.

35 Katon W, Cantrell CR, Sokol MC, Chiao E, Gdovin JM. Impact of antidepressant drug adherence on comorbid medication use and resource utilization. Arch Intern Med 2005; 165: 2497-503.

36 Scherrer JF, Garfield LD, Chrusciel T, Hauptman PJ, Carney RM, Freedland KE, et al. Increased risk of myocardial infarction in depressed patients with type 2 diabetes. Diabetes Care 2011; 34: 1729-34.

37 Gentil L, Vasiliadis HM, Preville M, Bosse C, Berbiche D. Association between depressive and anxiety disorders and adherence to antihypertensive medication in community-living elderly adults. J Am Geriatr Soc 2012; 60: 2297-301.

38 Burnett J, Coverdale JH, Pickens S, Dyer CB. What is the association between self-neglect, depressive symptoms and untreated medical conditions? J Elder Abuse Negl 2006; 18: 25-34.

39 Kisely S, Smith M, Lawrence D, Maaten S. Mortality in individuals who have had psychiatric treatment: population-based study in Nova Scotia. Br J Psychiatry 2005; 187: 552-8.

40 Fountoulakis KN, Veroniki AA, Siamouli M, Moller HJ. No role for initial severity on the efficacy of antidepressants: results of a multi-meta-analysis. Ann Gen Psychiatry 2013; 12: 26. 